\title{
Trayectorias escolares en el nivel medio superior: el caso de una institución pública
}

\author{
César Sánchez-Olavarría
}

\section{RESUMEN}

Este reporte de investigación busca caracterizar la trayectoria escolar de 513 estudiantes de una institución pública del nivel medio superior localizada en Tlaxcala, México, a partir del análisis de la estructura familiar, su eficiencia y el promedio. Se hallaron cuatro tipos de trayectorias escolares: óptima, aceptable, en rezago y en riesgo. La mayor parte de los estudiantes se ubicó en las trayectorias aceptable y óptima. Las trayectorias en rezago y en riesgo están constituidas principalmente por mujeres. El conocimiento de las problemáticas que enfrenta el estudiante durante su trayecto escolar permite a los docentes y directivos establecer estrategias de intervención para la acreditación y el trabajo en el aula.

Palabras clave: trayectorias escolares, educación media superior, estudiantes de educación media superior, estructura familiar, eficiencia, México.

\section{Trajetórias escolares no nível médio-superior: o caso de uma instituição pública}

\section{RESUMO}

Este relatório de pesquisa procura caracterizar a trajetória escolar de 513 estudantes de uma instituição pública do nível médio-superior localizada em Tlaxcala, México, a partir da análise da estrutura familiar, sua eficiência e a média do grupo. Se encontraram quatro tipos de trajetórias escolares: ótima, aceitável, com atraso e em risco. A maior parte dos estudantes se localizou nas trajetórias escolar aceitável e ótima. As trajetórias com atraso e em risco estão constituídas principalmente por mulheres. O conhecimento das problemáticas que enfrenta o estudante durante seu trajeto escolar permite aos docentes e diretivos estabelecer estratégias de intervenção para melhorar as notas e o trabalho na sala de aula.

Palavras chave: trajetórias escolares, ensino medio-superior, estudantes do ensino médio-superior, estrutura familiar, eficiência, México.

César Sánchez-Olavarría completo en el Posgrado en Educación de la Facultad de Ciencias de la Educación, UATX, México. Miembro del Sistema Nacional de Investigadores, nivel I. Temas de investigación: formación y mercado de trabajo; estudios de empleadores, trayectorias y egresados; desempeño y evaluación; competencias digitales y $b$-learning. 


\section{School Trajectories at Higher Secondary Level: the Case of a Public Institution}

\section{ABSTRACT}

This research report seeks to characterize the school trajectory of 513 students enrolled in a public institution at higher secondary level located in Tlaxcala, Mexico, based on the analysis of the family structure, its efficiency and the average. Four types of school trajectories were found: optimal, acceptable, lagging behind and at risk. Most students were in acceptable and optimal school trajectories. The lagging and at-risk trajectories concern mainly women. The knowledge of the problems faced by students during their school journey allows teachers and principals to establish intervention strategies for accreditation and classroom work.

Key words: school trajectories, high school, high school students, family structure, efficiency, Mexico. 


\section{Introducción}

El compromiso de las instituciones educativas con los estudiantes inicia desde el momento en que son aceptados los aspirantes de nuevo ingreso. Es tarea de las instituciones analizar los recorridos que van construyendo los estudiantes con la finalidad de llevarlos al otro lado del camino, es decir, al egreso. Éste es uno de los compromisos que adquieren las instituciones educativas, lo que impacta directamente en la disminución del rezago y la deserción y, por consecuencia, eleva la eficiencia terminal. Estos dos comportamientos académicos que ocurren en la vida escolar del estudiante reflejan una gran cantidad de problemas en la educación media superior. El rezago está compuesto por aquellos estudiantes que deben materias de semestres anteriores al que cursan o que están repitiendo alguna, por lo que su trayecto no tiene la misma regularidad que el de sus compañeros de generación. Esta situación es ocasionada por la influencia de factores personales tales como una enfermedad, divorcio de los padres, vivir en una familia disfuncional, consumo de drogas e ingesta de alcohol, el hecho de trabajar y estudiar, y aún la misma flojera y el poco interés por el estudio (March y Olsen, 1997; Fernández-Pérez, Peña-Chumacero y Vera-Rodríguez, 2006); de factores sociales como la influencia de los amigos, la escasa aceptación de sus pares, el bullying o el tener pareja (FernándezPérez, Peña-Chumacero y Vera-Rodríguez, 2006); o factores económicos, que se reflejan en los limitados recursos para la compra de útiles y libros, la transportación, las comidas y el material para realizar las tareas (Cuevas, 2001; Ponce de León, 2003).

Muchos de estos factores son desconocidos por la institución en la mayoría de los casos, lo que dificulta que la institución conozca el tipo de estudiante que se está formado y cuál ha sido su recorrido escolar. Contar con esta información contribuye a tomar decisiones fundamentadas para implementar estrategias de intervención dentro del ámbito académico, y canalizarlo al departamento correspondiente, de manera interna, o remitirlo con alguna institución especializada, de manera externa, con la finalidad de regularizar su situación escolar.

En lo que respecta a la deserción, de los países que integran la Organización para la Cooperación y el Desarrollo Económico (OCDE), México ocupa el primer lugar entre los jóvenes desertores de 15 a 18 años y es el tercer lugar de las naciones con mayor población juvenil que no estudia ni trabaja (OCDE, 2014). La obligatoriedad de la educación media superior (EMS), publicada en el Diario Oficial de la Federación el 9 de febrero de 2012 e implementada a partir del ciclo escolar 2012-2013, trajo consigo que los planteles de EMS aceptaran un mayor número de estudiantes. No obstante, este panorama excedía su capacidad de atención en detrimento de la calidad de su formación, debido a lo numeroso de los grupos y a la diversidad de capacidades de los estudiantes. Esta situación detonó las diferencias de las brechas de conocimiento entre los estudiantes de las distintas escuelas secundarias de origen, puesto que el nivel alcanzado en términos de habilidades, conocimientos y actitudes en cada una de ellas es muy distinto y los diversos factores externos presentes atenúan esas diferencias, a pesar de que en educación básica se manejan los mismos programas.

Estas dos problemáticas se reflejaron en la creación de políticas de retención a través de la implementación de programas de becas para la continuación de estudios, de transporte, de reinserción, de excelencia, de servicio social, de prácticas; programas para estudiantes con discapacidad, estímulos al talento o contra el abandono escolar, los cuales se ofertan de acuerdo con el estado, el subsistema y el plantel (SEP, 2017). Otra de las políticas está integrada por el movimiento contra el abandono escolar, el cual es una estrategia nacional en la EMS que involucra desde a los directivos del plantel hasta a los estudiantes y a la sociedad en general, con la finalidad de unir esfuerzos para la prevención del abandono en beneficio de la comunidad estudiantil (SEP, 2015). 
El estudio de estos comportamientos académicos está íntimamente conectado con un estudio de trayectorias escolares, puesto que es importante para las instituciones educativas obtener información sobre los recorridos que siguen los estudiantes con la finalidad de determinar qué tipo de trayectos se presentan, cómo se fueron construyendo, cuáles fueron los factores que incidieron en esos trayectos y establecer estrategias que incidan en la permanencia de los estudiantes (De Garay y Sánchez, 2012). Es responsabilidad de los planteles hacerse cargo de sus estudiantes desde su ingreso, durante su desempeño a través del programa educativo y hasta el egreso, por lo que la caracterización de los recorridos escolares se convierte en un insumo para las escuelas con la finalidad de elevar los índices de eficiencia terminal y así implementar acciones dirigidas a la resolución de las problemáticas identificadas. En este sentido, esta investigación pretende responder a las siguientes interrogantes: ¿Cuáles son las trayectorias escolares que se construyen en el nivel medio superior? ¿Cuáles son las características de las trayectorias escolares de los estudiantes del nivel medio superior, de acuerdo con la estructura familiar, eficiencia y promedio?; responder a ellas constituye el eje rector de la investigación.

$\mathrm{El}$ artículo está dividido en cuatro apartados: en el primero se retoman los orígenes de los estudios de trayectorias escolares, en el segundo se describe la estrategia metodológica empleada, en el tercero se da cuenta de los hallazgos de este estudio. Finalmente, el cuarto cierra con las conclusiones de la investigación.

\section{Antecedentes}

La contracción del mercado laboral y el creciente desempleo en los años setenta encienden las alarmas de las instituciones de educación superior (IES), lo que se refleja en la atención a esta problemática en dos vertientes: una vinculada con el exterior de la universidad y otra que se ocupa de lo que ocurre al interior de la misma. La primera buscaba obtener información acerca de los egresados y de los empleadores, con la intención de explicar lo que estaba ocurriendo en la arena laboral en cuanto al desempeño de los sujetos formados en la universidad; éste es el origen de los estudios de egresados y, más adelante, de los estudios de seguimiento de egresados, así como de los estudios de opinión de los empleadores en los noventa, con el auge de los procesos de calidad de las universidades y la acreditación de programas educativos (Sánchez-Olavarría, 2014).

Sin embargo, había otra preocupación relacionada con los procesos de formación de los sujetos dentro de las universidades. Esta segunda vertiente buscaba explicar las diversas problemáticas que enfrentan los estudiantes durante su recorrido escolar (Chaín-Revuelta, 1995). Se trataba de responder a los distintos problemas intraescolares que se presentan durante la interacción docente-estudiante y estudiante-estudiante, la construcción de aprendizajes, la enseñanza y la familia (De Allende y Gómez, 1989), los cuales se manifiestan en comportamientos específicos como la reprobación, la repetición, el rezago, el abandono, la eficiencia, la deserción y la eficacia (Chaín-Revuelta, 1995; Sánchez-Olavarría, 2014). La combinación de estos factores escolares con aspectos personales y socioeconómicos caracteriza trayectos exitosos, estancados, en rezago y no exitosos, sobre todo en la educación superior (Chaín-Revuelta, 1995; Chaín-Revuelta, Jácome y Martínez, 2001; Cuevas, 2001; Fernández-Pérez, Peña-Chumacero y Vera-Rodríguez, 2006; Terigi, 2007; De Garay y Sánchez, 2012; Sánchez, Alamilla y García, 2013), donde se desarrollan básicamente este tipo de trabajos. Sin embargo, ya existen estudios de trayectoria escolar que abordan otros niveles educativos (González, 1999; Fernández-Pérez, Peña-Chumacero y Vera-Rodríguez, 2006).

\section{Hacia una definición de trayectoria escolar}

La deserción, el rezago, el abandono, la reprobación, el rendimiento escolar y la eficiencia terminal son algunos de los temas recurrentes que hacen alusión al 
comportamiento escolar de los estudiantes durante su paso por los diferentes niveles educativos en los que transita. Es común encontrar investigaciones que abordan algunos de estos aspectos de manera aislada, sin embargo, los estudios de trayectorias escolares integran estas problemáticas a fin de analizar los factores que inciden en la construcción de determinado recorrido.

El análisis de la información recabada ha permitido identificar tres posturas respecto a los estudios de trayectorias escolares en la actualidad: una relacionada con el comportamiento de los estudiantes durante su recorrido por un determinado nivel educativo, otra relativa a los factores de influencia que provocan que un individuo construya tal o cual trayecto de formación, y una más que incluye tanto los factores como el comportamiento. El análisis de estas rutas de formación ayuda a las autoridades a establecer programas de prevención como la tutoría, como una forma de atención y seguimiento personalizada para canalizar a los estudiantes que así lo requieran, en lo que concierne a conocimientos, habilidades y actitudes (Badillo, 2007). La primera postura hace alusión a los recorridos institucionales que desarrollan los estudiantes en términos de la regularidad/irregularidad académica del trayecto, el cual es establecido por el sistema educativo (Altamira, 1997; Barranco y Santacruz, 1995). Este tipo de trayectoria va asociada a cuestiones de deserción, rendimiento, reprobación, rezago y eficiencia terminal. En la segunda perspectiva se trabaja con información acerca de la familia de origen, la trayectoria previa y el capital social como factores que influyen en el ingreso, en su permanencia y hasta el egreso, lo que caracteriza un determinado trayecto formativo (March y Olsen, 1997; González, Castro y Bañuelos, 201 1). La tercera postura integra el análisis del comportamiento de un grupo de estudiantes, lo que construye una determinada ruta de formación que está caracterizada por factores internos y externos (Fernández-Pérez, Peña-Chumacero y Vera-Rodríguez, 2006; Cuevas,
2001; Ponce de León, 2003; Chaín-Revuelta. Y Ramírez-Muro, 1997).

Para efectos de la presente investigación, las trayectorias escolares se conceptualizan como el análisis de los factores personales, sociales y académicos que determinan el comportamiento de un grupo de estudiantes durante su tránsito por un nivel educativo

\section{Dimensiones de análisis}

En la trayectoria escolar se trabajan tres dimensiones: temporalidad, eficiencia y rendimiento. La primera analiza el recorrido del estudiante por el nivel educativo en el que se encuentra. Estos trayectos pueden ser continuos (Chaín-Revuelta, 1995) o teóricos (Terigi, 2007), los cuales están determinados por los tiempos estándar establecidos por la institución para que un estudiante promedio cumpla con la obtención de créditos señalada para cada uno de los semestres cursados. Se hace referencia al recorrido regular que sigue un estudiante durante su estancia en un nivel educativo determinado, el cual está vinculado a su generación. Es el periodo de tiempo habitual que tarda un estudiante en realizar un trayecto escolar en un nivel educativo.

No obstante, existen trayectos que no tienen la regularidad de los anteriores, los cuales se denominan discontinuos (Chaín-Revuelta, 1995) o reales (Terigi, 2007). Este tipo de recorridos son irregulares, se presenta en los estudiantes durante su trayectoria escolar y se evidencia con el recursamiento de materias, lo que se traduce en rezago en comparación con los estudiantes de su generación. El trayecto discontinuo es el periodo o periodos extra que toma un estudiante para la obtención de créditos que lo llevan a concluir el nivel educativo en un momento posterior al de su generación. Chaín-Revuelta, Jácome y Martínez (2001) analizan esta dimensión y proponen una trayectoria continua o discontinua, a partir de la aprobación o no aprobación del total de cursos tomados. De Garay y Sánchez (2012) clasifican una trayectoria en regular, irregular y en rezago a partir 
del porcentaje de créditos obtenidos durante el primer año de estudios de la carrera.

La eficiencia constituye la segunda dimensión y se refleja en cómo los estudiantes aprueban las materias, en términos del periodo de evaluación en el que el alumno pasa una materia o conjunto de materias determinadas. Se presenta en tres momentos: ordinaria, no ordinaria e irregular (Chaín-Revuelta, 1995). La primera se muestra cuando el estudiante aprueba sus materias al finalizar el periodo normal mínimo establecido (semestre, cuatrimestre, trimestre o ciclo) por la institución, es decir, en su primera oportunidad. La segunda se relaciona con la aprobación de materias en examen extraordinario, a título de suficiencia o en recursamiento, pero dentro del mismo ciclo escolar de su generación. Finalmente, la tercera se relaciona con tomar materias en un turno diferente al que está integrado o en un periodo distinto al de su generación, lo que se constituiría en una trayectoria en rezago. Por medio del porcentaje de asignaturas aprobadas en evaluación ordinaria, Chaín-Revuelta,Jácome y Martínez (2001) clasifican una trayectoria de este tipo en baja, media y alta.

La tercera dimensión es el rendimiento, el cual se obtiene en dos momentos: durante las evaluaciones parciales y al final del ciclo. En el primer caso permite realizar ajustes intermedios en el proceso de aprendizaje de los estudiantes con la intención de mejorar durante el ciclo. El segundo se obtiene con el promedio final que se consigue de la suma de las calificaciones obtenidas en las materias cuando termina el ciclo y divididas entre el número de materias cursadas. Esta dimensión es utilizada en el estudio realizado por Sánchez, Alamilla y García (2013), en el que combina la eficiencia con el promedio para establecer una nueva tipología: alumnos regulares excelentes, regulares, irregulares, irregulares bajos y egresados titulados.

La revisión de literatura acerca de los estudios de trayectoria ha permitido identificar una diversidad de trabajos, los cuales se pueden agrupar en cuatro posturas: los que analizan recorridos laborales de los sujetos (trayectorias laborales y trayectorias ocupacionales), los que trabajan el tránsito profesional (trayectorias profesionales), los que abordan aspectos de formación (trayectorias de participación, trayectorias educativas, trayectorias académicas y trayectorias escolares) (Sánchez-Olavarría, 2017) y los que son de tipo transversal que contribuyen con los tres anteriores para dar una explicación del porqué de un trayecto determinado (trayectorias personales, trayectorias familiares y trayectorias juveniles). En esta investigación se trabajó en la caracterización de las trayectorias escolares de los estudiantes de una institución pública del nivel medio superior.

Existe una serie de fundamentos teóricos económicos y sociológicos que ha fundamentado la interpretación de los diversos caminos que un individuo va construyendo y que conforma su trayectoria. El capital cultural de Bourdieu se ha utilizado para tratar de explicar las trayectorias escolares de los estudiantes, puesto que se resalta la importancia de las relaciones interpersonales que van creando los sujetos durante su vida. La familia, los vínculos sociales que construyen y su formación establecen diferencias que dan origen a la creación de estructuras sociales. Bourdieu (2000) señala que la cultura y la formación académica son factores que favorecen a la distinción entre las personas, la cual no existe más que por una oposición al otro, ese rasgo distintivo diferenciador entre los sujetos. La distinción es el elemento base para la constitución y la preservación de los espacios sociales en los que se desarrolla cierto tipo de personas.

El estado incorporado del capital cultural de una persona se manifiesta en la creación de redes interpersonales que se manifiestan en la realización de prácticas sociales comunes, las cuales se pueden oponer a otras a partir de los principales principios de diferenciación: el capital económico y el capital cultural. Bourdieu (1990) enfatiza que este último principio es uno de los factores que está directamente relacionado con el éxito o fracaso escolar. El capital 
cultural desarrollado por una familia en su estado incorporado (condiciones intrínsecas y relacionales de las personas), subjetivado (bienes culturales concretos y abstractos) e institucionalizado (credenciales obtenidas) condiciona la trayectoria de sus integrantes. "Lo que el obrero come y sobre todo su manera de comerlo, el deporte que practica y su manera de practicarlo, sus opiniones políticas y su manera de expresarlas difieren sistemáticamente del consumo o de las actividades correspondientes del industrial" (Bourdieu, 2000: 33).

$\mathrm{El}$ análisis de los factores que inciden en las trayectorias escolares en los diferentes niveles educativos, en los que predomina la educación superior, ha permitido caracterizar los recorridos tomando en cuenta alguno de ellos o la combinación de varios. En un estudio realizado por Chaín-Revuelta, Jácome y Martínez (2001), se trabajó con una muestra del 30\% de los estudiantes que ingresaron a la Universidad Veracruzana en la generación 1998-2002, durante los tres primeros semestres. Se siguió un enfoque cuantitativo a partir de técnicas de inteligencia artificial llamadas "descubrimiento de conocimiento en bases de datos" y la aplicación de algoritmos de Minería de Datos. Se aplicó un cuestionario de 38 reactivos. Se reconstruyeron sus trayectorias a partir de una tipología que consideró cuatro elementos: continuidad (si el estudiante ya cuenta con los créditos correspondientes en el momento que se encuentra su generación); aprobación (cursos aprobados en primera oportunidad); eficiencia (relación entre los cursos inscritos con los cursos promovidos) y rendimiento (obtenido por el estudiante). La combinación de estos elementos con la trayectoria previa se tradujo en la obtención de 18 trayectorias, de las cuales se destacó que el 16\% de los estudiantes construyeron una trayectoria discontinua baja con recorrido previo bajo, otro $16 \%$ desarrolló una trayectoria discontinua baja con recorrido previo medio y un $13 \%$ construyó una trayectoria continua alta con recorrido previo alto. El 55\% restante se repartió en los 15 trayectos faltantes.
De Garay y Sánchez (2012) analizaron los cambios ocurridos en las trayectorias escolares de dos generaciones (2003 y 2008) de estudiantes de las divisiones académicas de Ciencias Básicas e Ingeniería (CBI), Ciencias Sociales y Humanidades (CSH) y Ciencias y Artes para el Diseño (CyAD) de la Universidad Autónoma Metropolitana (UAM) Azcapotzalco durante el primer año de estudios. Se reprodujo un cuestionario, validado y aplicado en un estudio financiado por la ANUIES en el 2000, construido a partir de las siguientes dimensiones: perfil socioeconómico y cultural, hábitos de estudio, opinión sobre sus profesores, prácticas de consumo cultural y expectativas de desarrollo futuro profesional y académico. Este instrumento se aplicó a todos los estudiantes en dos momentos: al inscribirse por primera vez y al terminar el año escolar. Se identificaron tres tipos de trayectoria en cada generación: regular (estudiantes con más del 90\% de los créditos), irregular (estudiantes entre el 50 y $89 \%$ de los créditos) y en rezago (menos del $49 \%$ de los créditos). Al realizar un comparativo entre ambas generaciones, se encontró que se incrementaron las trayectorias regulares en la generación 2008 en cinco puntos. Las trayectorias irregulares se mantuvieron estables con una diferencia de un punto, pero las trayectorias en rezago disminuyeron cuatro puntos en la generación 2008. Por divisiones, CBI y CSH incrementaron su regularidad en la generación 2008 en siete puntos en promedio y CyAD la disminuyó en casi dos puntos.

Sánchez, Alamilla y García (2013) estudiaron los principales factores que afectan las trayectorias escolares de los estudiantes inscritos en la licenciatura de Derecho y Jurisprudencia del Instituto de Ciencias Sociales y Humanidades de la Universidad Autónoma del Estado de Hidalgo. Realizaron un diseño de investigación no experimental, transversal, en el que aplicaron un cuestionario. Este instrumento estuvo constituido por cuatro apartados: datos generales, formación escolar previa, condiciones socioeconómicas y factores de influencia en las trayectorias. Se 
piloteó y validó (alfa de Cronbach 0.90). Se trabajó con una muestra aleatoria del 10\% (72 estudiantes) de las generaciones de 2006 a 2011. Se identificaron cuatro tipos de trayectorias: regulares excelentes (alumnos que no deben ninguna materia y tiempo promedio de 9 a 10), regulares (que no deben materias y promedio menor a 9), irregulares (que deben una materia) e irregulares bajos (que deben dos o más materias). Se encontró que el 50\% desempeñó recorridos regulares y el otro $50 \%$ construyó recorridos irregulares, de los cuales el 15\% de los estudiantes lograron una trayectoria regular excelente, el 35\% se desenvolvió en recorridos regulares, 19\% obtuvo trayectorias irregulares y el restante $31 \%$ fue construyendo recorridos irregulares bajos.

\section{Estrategia metodológica}

El plantel en el que se realizó la investigación es una institución pública del nivel medio superior de modalidad educativa presencial escolarizada que se fundó en 1982 y empezó funciones con una población de 269 estudiantes, 17 docentes y siete administrativos. En la actualidad cuenta con 49 docentes entre técnicos, ingenieros y licenciados $4 \%$ con doctorado y $22 \%$ con maestría). Se imparten cinco especialidades: Mantenimiento Automotriz, Contabilidad, Recursos Humanos, Programación y Diseño de Modas. La principal actividad de la población en la que se ubica el plantel se desarrolla en la industria manufacturera y construcción. Los padres de este grupo de estudiantes se desempeñan como comerciantes $(22 \%)$, empleados textiles $(20 \%)$, albañiles $(20 \%)$, choferes de transporte público (17\%), profesionistas (12\% - contador, profesor, abogado, arquitecto-), $\operatorname{artesanos}(6 \%)$ y en el hogar $(3 \%)$. En lo que concierne a las madres, el 55\% se desempeña como ama de casa, el 20\% trabaja en talleres de costura, el 10\% es comerciante y el $15 \%$ es asistente.

La presente investigación muestra parte de los resultados obtenidos de un estudio de trayectorias escolares realizado en una institución de educación media superior ubicada en Tlaxcala, México y tuvo como objetivo caracterizar las trayectorias escolares de los estudiantes del plantel ya mencionado. Es un estudio descriptivo-transversal, en el que se recolectó la información en un solo momento. La población estuvo constituida por 1262 estudiantes (ver tabla 1), divididos en 27 grupos (15 matutino y 12 vespertino), de los cuales se tomó una muestra intencionada de la mitad de los grupos, 8 del turno matutino y 6 del vespertino, lo que dio como resultado 513 estudiantes. Una de las razones por las que se trabajó con una muestra fue que se contaba con recursos limitados. Sin embargo, se tenía la necesidad de realizar un diagnóstico de la situación en que se encontraban los estudiantes al inicio del ciclo escolar, por lo que se buscó que la muestra representara ambos turnos y especialidades para obtener un panorama general de la población y diseñar una estrategia de intervención para reducir la reprobación y elevar la eficiencia terminal del plantel. Los sujetos de investigación estaban constituidos por el $56 \%$ de mujeres y el $44 \%$ de varones, distribuidos en sexto, 137 $(27 \%)$; cuarto, 145 (28\%), y segundo, 231 (45\%).

La recolección de información se realizó a partir de un cuestionario que consta de 56 reactivos, dividido en cuatro secciones: datos sociodemográficos (género, edad y semestre), estudios previos (promedio y secundaria de origen), condiciones socioeconómicas (grado máximo de estudios de los padres, ingresos y estructura familiar) y factores que intervienen en la trayectoria escolar (rendimiento, número de materias reprobadas, semestre con mayor reprobación). Este instrumento fue validado por un grupo de expertos en la temática abordada. Posteriormente, se piloteo con dos grupos de estudiantes del nivel medio superior con la finalidad de revisar el tiempo de aplicación, la claridad de las preguntas y las respuestas. Se obtuvo un Alpha de Cronbach de 0.84. Se tomaron en cuenta las observaciones realizadas para elaborar la versión final del cuestionario y se procedió a aplicarlo. Para el análisis de la información se utilizó el software SPSS versión 21. 
Tabla 1. Distribución de la población por especialidad

\begin{tabular}{|l|c|c|c|}
\hline & Matutino & Vespertino & Total \\
\hline Programación & 207 & 116 & 323 \\
\hline Contabilidad & 89 & 0 & 89 \\
\hline Recursos humanos & 0 & 75 & 75 \\
\hline Diseño de modas & 88 & 27 & 115 \\
\hline Mantenimiento automotriz & 87 & 0 & 87 \\
\hline Componente básico & 293 & 280 & 573 \\
\hline Total & $\mathbf{7 6 4}$ & $\mathbf{4 9 8}$ & $\mathbf{1 2 6 2}$ \\
\hline
\end{tabular}

Fuente: elaboración propia.

\section{Resultados}

La edad de los estudiantes fluctúa entre los 15 y los 19 años con un promedio de 17. Dentro de la trayectoria escolar de los estudiantes de bachillerato en el nivel educativo que les precede, se encontró que la mayoría de los estudiantes procede de secundarias técnicas y generales, $61 \%$ y $32 \%$ respectivamente (ver gráfica 1). El porcentaje restante proviene de telesecundarias $(5 \%)$ y secundarias particulares $(2 \%)$. El $46 \%$ de la población cuenta con algún tipo de beca, ocupando "PROSPERA" el 37\%; 54\% no tiene beca, $4 \%$ beca SEP y $5 \%$ beca económica.

La institución se encuentra ubicada en la comunidad de Santa Cruz Guadalupe. El 80\% de la población proviene de cinco municipios del estado de Tlaxcala (ver gráfica 2): Santa Ana Chiautempan (42\%), San Bernardino Contla (18\%), La Magdalena Tlaltelulco $(10 \%)$, San Pablo Apetatitlán $(5 \%)$ y San Luis Teolocholco (5\%). Tres de esos municipios se encuentran ubicados a una distancia promedio de $4 \mathrm{~km}$ del plantel (Chiautempan, Contla y Apetatitlán) y los dos municipios restantes se ubican a $12 \mathrm{~km}$ de la institución. Es importante señalar que a una distancia muy cercana de estos dos últimos municipios se ubican tres instituciones de educación media superior, CBTa 134, COBAT 16 y CECyTE 16, no obstante, los estudiantes deciden estudiar en el CEtis, principalmente por sus especialidades.

La mayor proporción en lo que se refiere a la escolaridad del padre se concentra en el nivel básico (41\% secundaria y $16 \%$ primaria), seguido con un distante $29 \%$ del nivel bachillerato, $11 \%$ del nivel licenciatura y un escaso nivel de posgrado (3\%). En lo que respecta a la escolaridad de las madres, éstas se ubican principalmente en el nivel básico ( $55 \%$ secundaria y sólo el $1 \%$ con primaria), seguido del nivel bachillerato $(30 \%)$, $13 \%$ del nivel licenciatura y prácticamente nulo nivel de posgrado $(1 \%)$. La escolaridad de ambos padres (ver tabla 2) se ubica por debajo de los niveles medio superior y superior, lo que significa que el estado institucionalizado de su capital cultural (Bourdieu, 2000) no es suficiente y dificulta su labor en términos de la ayuda académica que pudieran ofrecer a sus hijos, por lo que se presenta una tendencia a reproducir los patrones seguidos por los padres. En promedio, se encontró que poco más de la mitad de los estudiantes reportaron que sus padres contaban con estudios de nivel básico. Este resultado confirma los hallazgos de otros investigadores (García y Barrón, 2011; González, Castro y Bañuelos, 2011), quienes han mostrado que la escolaridad de la mayoría de los padres que tienen hijos en el nivel medio superior o superior se ubica en el nivel básico. 


\section{Grafica 1. Secundaria de procedencia}

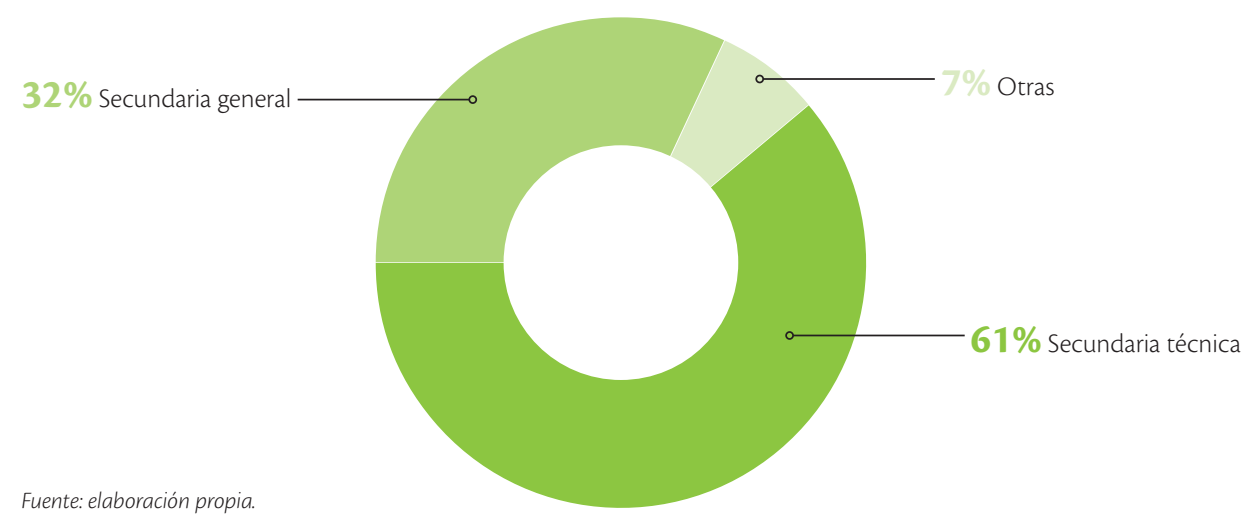

Gráfica 2. Municipios de procedencia

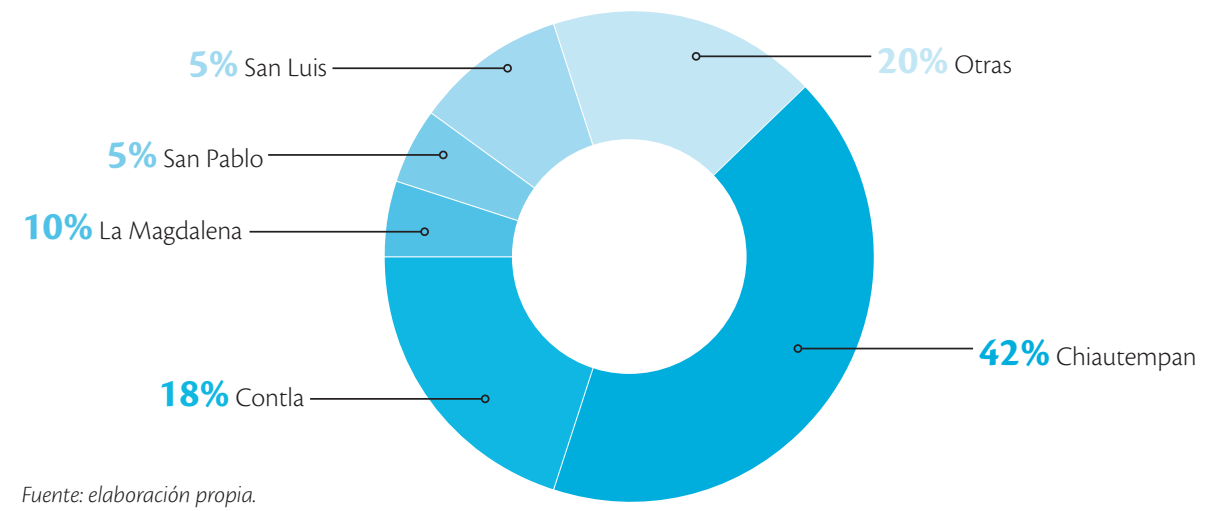

Tabla 2. Escolaridad de los padres

\begin{tabular}{|l|c|c|}
\hline \multicolumn{1}{|c|}{ Nivel educativo } & Padre \% & Madre \% \\
\hline Doctorado & 1 & 0 \\
\hline Maestría & 2 & 1 \\
\hline Licenciatura & 11 & 13 \\
\hline Bachillerato & 29 & 30 \\
\hline Secundaria & 41 & 55 \\
\hline Primaria & 16 & 1 \\
\hline Total & $\mathbf{1 0 0}$ & $\mathbf{1 0 0}$ \\
\hline
\end{tabular}

Fuente: elaboración propia. 
La trayectoria previa de los estudiantes estuvo constituida por el rendimiento académico alcanzado en el nivel básico (ver gráfica 3), en el que se encontró que la proporción más alta se concentra entre los estudiantes que obtuvieron promedios entre 8.1 y 9 (44\%), seguida por aquellos que consiguieron promedios entre 7.1 y $8(35 \%)$, lo que constituye el $79 \%$ de la muestra estudiada. El 16\% logró un promedio entre 9.1 y 10 y una mínima proporción obtuvo un rendimiento entre 6 y $7(5 \%)$. En lo que respecta al nivel medio superior, se identificó una alta concentración de estudiantes en el rango de 7.1 y $8(46 \%)$. El segundo rango con mayor presencia de estudiantes fue el promedio entre 8.1 y $9(28 \%)$. Estos resultados concuerdan con lo encontrado por De Garay y Sánchez (2012) y García y Barrón (2011) en relación con que éste es el rango que se presenta con mayor frecuencia en los promedios obtenidos en el nivel medio superior. En menor proporción se ubicaron los promedios entre 6 y $7(19 \%)$ y entre 9.1 y $10(7 \%)$. A pesar de que hubo coincidencia en cuanto a que la mayor cantidad de estudiantes se concentró en dos rangos de promedios, 7.1 y 8 y 8.1 y 9 en ambos niveles educativos, es evidente que en bachillerato se presentó una disminución en los promedios altos de entre 9 y 26 puntos porcentuales y, por consiguiente, un aumento en los promedios bajos de entre 11 y 14 puntos, respectivamente. Es importante destacar este hecho, puesto que dos de los principales planes a futuro de los estudiantes son estudiar una licenciatura $(63 \%)$ y realizar estudios de posgrado, maestría y doctorado (37\%), sin embargo, sólo el 35\% de estos jóvenes tiene promedio de 8.1 o mayor, uno de los requisitos principales para la obtención de una ficha en la mayoría de la universidades en Tlaxcala.

El 51\% de la población estudiada refirió que no ha reprobado materia alguna durante su recorrido por el bachillerato, no obstante, la reprobación se mantiene como una situación recurrente en EMS y uno de los principales factores que originan la deserción. La SEP (2016), en el documento denominado "Principales cifras del sistema educativo nacional 2015-216", muestra que dentro de la educación obligatoria, la EMS presenta el mayor índice de reprobación en los últimos dos años con un promedio de 15.8\%. El 39\% de los estudiantes ha reprobado una o dos materias y el 10\% entre tres o cuatro (ver gráfica 4). El mayor índice de reprobación se presenta en el primer semestre $(48 \%)$, seguido del segundo semestre (29\%), lo cual indica que este fenómeno ocurre durante el primer año de bachillerato $(77 \%)$. Los estudiantes de bachillerato indicaron, por orden descendente, que las tres materias problemáticas son inglés $30 \%$, química $23 \%$ y matemáticas 19\%, las cuales presentan los niveles de reprobación más altos.

\section{Gráfica 3. Comparativo por promedios en secundaria y bachillerato}

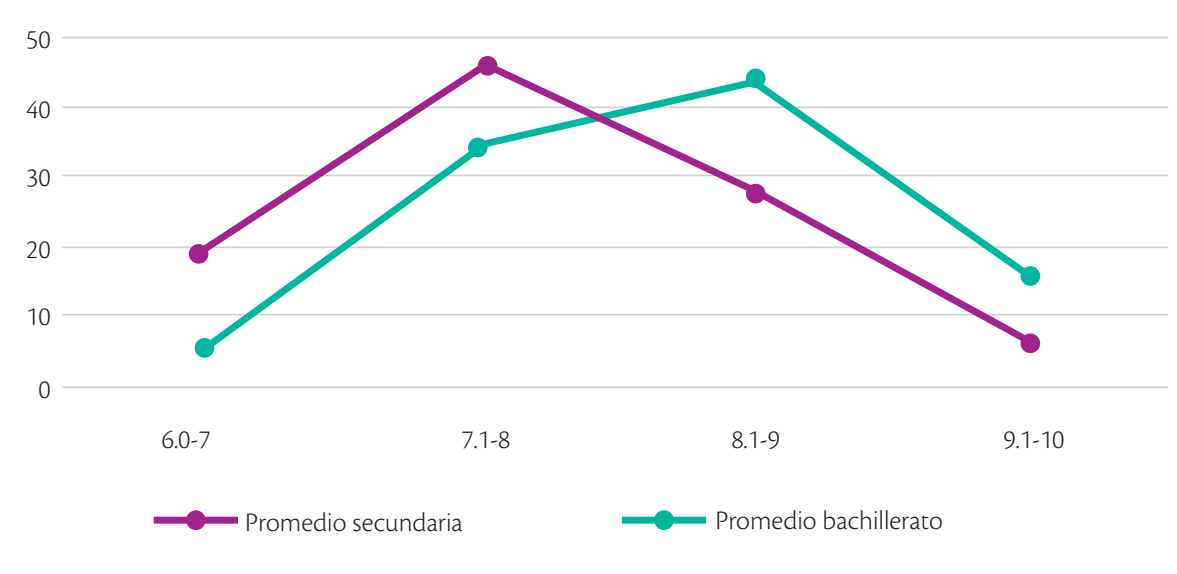

Fuente: elaboración propia. 


\section{Gráfica 4. Número de materias reprobadas en EMS}

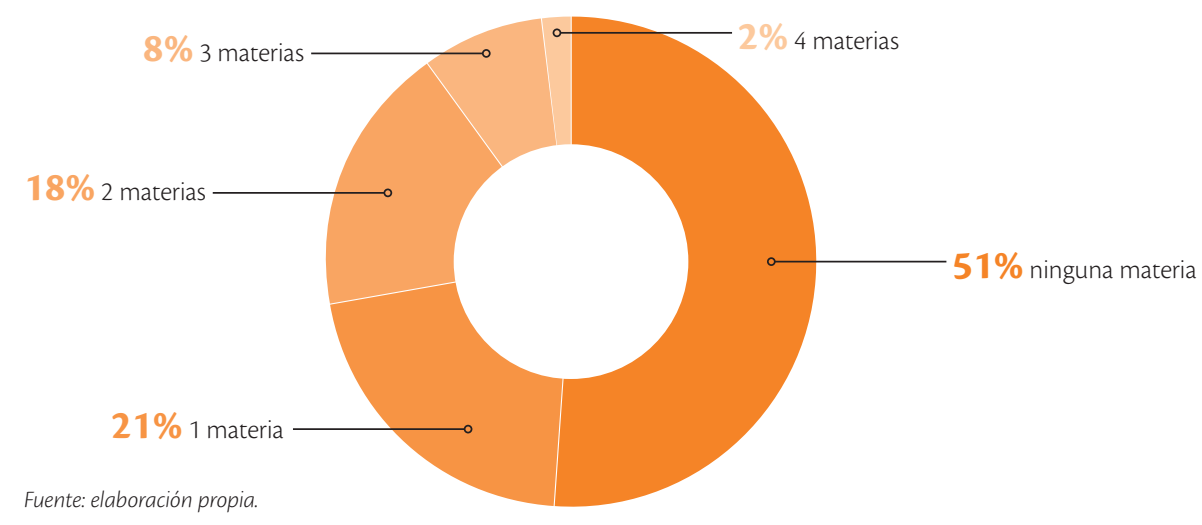

\section{Trayectorias escolares en el nivel medio superior}

Para la integración de las trayectorias, se diseñó una síntesis combinatoria entre estructura familiar, eficiencia y promedio. Para la estructura familiar se consideraron la funcional y la disfuncional. La primera abarca a los estudiantes que viven con ambos padres. La segunda considera a los estudiantes que sólo viven con la madre o con el padre. Ambas estructuras abarcan el $96 \%$ de la muestra, por lo que no se tomaron en cuenta los otros dos tipos de familia: los que viven en pareja y los que viven con tíos o abuelos, puesto que en conjunto sólo contemplan el 4\%. Para el caso de la eficiencia, la ordinaria está definida para los estudiantes que no deben ninguna materia y la no ordinaria se constituye por aquellos que han reprobado o que deben alguna materia. En lo que respecta al promedio, éste fue dividido en cuatro grupos: muy alto (9.1-10), alto (8.1-9), regular (7.1-8) y bajo (6-7).

La integración de estos tres factores permitió identificar cuatro tipos de trayectoria: trayectoria óptima (TO), trayectoria aceptable (TA), trayectoria en rezago (TRE) y trayectoria en riesgo (TRI) (ver tabla 3). Para este análisis sólo se consideraron las trayectorias con porcentajes del $5 \%$ o mayores en su rendimiento académico. La trayectoria óptima es realizada por el $20 \%$ de la muestra encuestada. Se caracteriza porque el estudiante proviene de una familia funcional, en la que los padres cuentan con estudios de licenciatura y en menor medida de posgrado, no ha reprobado alguna materia y ha desarrollado un rendimiento académico alto o muy alto. Esta trayectoria presenta una pequeña diferencia a favor de los varones, puesto que éstos constituyen el $11 \%$ y las mujeres el $9 \%$. La trayectoria aceptable está compuesta por el $40 \%$ de la población. Los padres han cursado estudios universitarios en un porcentaje bajo, ya que la mayoría tiene el bachillerato y un número reducido cuenta con secundaria. Se caracteriza por presentarse en cifras similares en ambos sexos, principalmente en estudiantes con una familia funcional $(34 \%)$ y sólo el $6 \%$ proviene de estudiantes con familias disfuncionales. A pesar de que en esta trayectoria no se reprueba materia alguna, su rendimiento académico está por debajo de la trayectoria óptima, puesto que su desempeño es regular o bajo. En promedio, los porcentajes en estos dos tipos de trayectoria son muy semejantes entre varones y mujeres, lo que contrasta con el estudio realizado por De Garay y Sánchez (2012), quienes señalan que las trayectorias regulares (positivas) son construidas principalmente por mujeres, debido a su mayor compromiso y fortaleza ante las dificultades que enfrentan. 
La trayectoria en rezago se compone por estudiantes del sexo femenino que provienen de una familia funcional, en la que los padres cuentan con estudios de bachillerato y secundaria en porcentajes similares. Han reprobado alguna materia, es decir, presentan una eficiencia no ordinaria y promedios alto y muy alto $(20 \%)$. Finalmente, la trayectoria en riesgo está compuesta por estudiantes mujeres con familias disfuncionales $(5 \%)$, con eficiencia no ordinaria, que han reprobado materias o deben alguna de ellas y promedios bajo y regular. Los padres de familia sólo tienen estudios de educación básica (secundaria y primaria). Los estudiantes (mujeres o varones), que provienen de una estructura familiar en la que están presentes el padre y la madre y que consiguieron una eficiencia ordinaria, desarrollaron recorridos positivos, los cuales se reflejaron en la construcción de trayectorias óptimas y aceptables. Existe otro grupo de estudiantes (sólo mujeres) que desarrollaron recorridos negativos, los cuales tienen dos orígenes: aquellas que cuentan con una estructura familiar funcional con eficiencia no ordinaria (TRE), con promedios regulares y bajos principalmente y algunas con promedios altos y las que provienen de una estructura disfuncional con eficiencia no ordinaria (TRI) y promedios bajos.

Las trayectorias positivas $(54 \%$ entre óptima y aceptable) se caracterizaron por un capital cultural mayor que las trayectorias negativas (en rezago y en riesgo). El estado incorporado (relaciones interpersonales de mayor calidad al interior del núcleo familiar, al constituirse en familias funcionales y con un mayor nivel cultural) y el estado institucionalizado (mayor nivel de estudios de los padres desde bachillerato hasta posgrado) del capital cultural que los estudiantes construyeron, reflejaron trayectorias exitosas, lo que confirma lo dicho por Bourdieu (2000) respecto a que la cultura y la formación académica de los padres son factores que reducen la posibilidad del fracaso escolar, debido a la preparación, atención, comunicación y compromiso con los hijos.

Tabla 3. Trayectorias en porcentajes

\begin{tabular}{|c|c|c|c|c|c|c|c|}
\hline \multirow{2}{*}{$\begin{array}{c}\text { Género } \\
\text { Hombre } 43\end{array}$} & \multirow{2}{*}{$\begin{array}{c}\begin{array}{c}\text { Estructura } \\
\text { familiar }\end{array} \\
\text { Funcional-F } \\
\text { Disfuncional-D }\end{array}$} & \multirow{2}{*}{$\begin{array}{c}\text { Eficiencia } \\
\text { Ordinario-O } \\
\text { No ordinario-N }\end{array}$} & \multicolumn{4}{|c|}{ Promedio } & \multirow{2}{*}{$\begin{array}{c}\begin{array}{c}\text { Tipo de } \\
\text { Trayectoria }\end{array} \\
\text { TO/TA } \\
\text { TRE/TRI }\end{array}$} \\
\hline & & & $\begin{array}{l}6-7 \\
\text { Bajo }\end{array}$ & $\begin{array}{c}\text { 7.1-8 } \\
\text { Regular }\end{array}$ & $\begin{array}{l}8.1-9 \\
\text { Alto }\end{array}$ & $\begin{array}{c}9.1-10 \\
\text { Muy alto }\end{array}$ & \\
\hline & \multirow[t]{2}{*}{ F 36} & O 31 & 5 & 15 & 9 & 2 & TA/TO \\
\hline & & N 5 & 1 & 2 & 1 & 1 & \\
\hline & \multirow[t]{2}{*}{ D 07} & O 1 & 0 & 1 & 0 & 0 & \\
\hline & & Mujer 57 & 1 & 3 & 2 & 0 & \\
\hline \multirow[t]{3}{*}{ Mujer 57} & \multirow[t]{2}{*}{ F 43} & O 23 & 4 & 10 & 6 & 3 & TA/TO \\
\hline & & N 20 & 4 & 9 & 6 & 1 & TRE \\
\hline & \multirow[t]{2}{*}{ D 14} & O9 & 2 & 4 & 2 & 1 & TA \\
\hline$N=513$ & & N 5 & 2 & 3 & 0 & 0 & TRI \\
\hline Totales & 100 & 100 & 18 & 47 & 27 & 8 & \\
\hline
\end{tabular}

Fuente: elaboración propia. 


\section{Conclusiones}

El conocimiento de las problemáticas que enfrenta el estudiante durante su trayecto escolar por la educación media superior permite a los docentes y directivos establecer estrategias de intervención en dos sentidos: para la acreditación en términos del cumplimiento de indicadores que aseguren la "calidad" de los programas educativos y para la implementación de programas de atención que impacten en el aula. El estudio de la trayectoria escolar en los estudiantes de bachillerato es uno de los indicadores de la categoría 8 (Servicios Escolares) que deben cubrir los planteles para ingresar o promocionarse en el Sistema Nacional de Bachillerato (SNB), por lo que su realización es obligatoria para la acreditación del plantel por un organismo externo reconocido por el Consejo para la Evaluación de la Educación del Tipo Medio Superior (COPEEMS), lo que se traduce en un proceso eficientista y mecanizado (Silas, 2013).

Sin embargo, más allá de la utilidad que tiene este tipo de estudios a nivel institucional en términos del cumplimiento de indicadores para la obtención de uno de los niveles del SNB, la información que se obtiene contribuye con la identificación de problemáticas específicas en la vida estudiantil de un determinado grupo de estudiantes de cierto plantel educativo. La importancia de esta segunda vertiente radica en el análisis de los datos recabados y la consecuente obtención de información para la implementación de estrategias de prevención y programas remediales (González, 2000; Gutiérrez, Granados y Landeros, 2011; De Garay y Sánchez, 2012) en un primer momento, pero también en la mejora de los procesos de formación y potenciar sus conocimientos, habilidades y actitudes. La información arrojada por un estudio de trayectorias escolares permite conocer las condiciones de acceso a un nivel educativo, lo que contribuye con datos (socioeconómicos, personales y académicos) para la planeación y para seguir de cerca el recorrido académico de los estudiantes e implementar estrategias de intervención con la finalidad de elevar la retención y, por consecuencia, la eficiencia terminal de las instituciones (INEE, 2012). El origen familiar, en este caso familias disfuncionales, los bajos niveles de escolaridad de los padres, el limitado ingreso económico y el género fueron los principales factores que determinaron los comprometidos recorridos escolares (TRE y TRI) de los estudiantes de esta institución de educación media superior. El hecho de que en este tipo de trayectorias se ubique a cierto número de mujeres es un hallazgo contrario a otras investigaciones, en las que ellas muestran mayor disposición y fortaleza ante la adversidad (De Garay y Sánchez, 2012). 


\section{Referencias}

Altamira, A. (1997), "El análisis de las trayectorias escolares como herramienta de evaluación de la actividad académica universitaria: un modelo ad hoc para la Universidad Autónoma de Chiapas, el caso de la Escuela de Ingeniería Civil”, Tesis de maestría inédita, Tuxtla, Universidad Autónoma de Chiapas.

Badillo, J. (2007), "La tutoría como estrategia viable de mejoramiento de la calidad de la educación superior. Reflexiones en torno al curso", en CPU-e, Revista de Investigación Educativa, núm. 5, pp. 1-22, <http://www. redalyc.org/pdf/2831/283121712006.pdf> [Consulta: octubre de 2015].

Barranco, S., M. y M. C. Santacruz (1995), Los egresados de la UAA. Trayectoria escolar y desempeño laboral, México, Universidad Autónoma de Aguascalientes.

Bourdieu, P (2000), Capital cultural, escuela y espacio social, México, Siglo XXI.

Bourdieu, P (1990), Sociología y cultura, México, Grijalbo/CONACULTA.

Chaín-Revuelta, R. (1995), "Estudiantes universitarios. Trayectorias escolares en la Universidad Veracruzana", México, Universidad Autónoma de Aguascalientes, <https://www.uv.mx/cpue/colped/N_2526/ publragu.htm $>$ [Consulta: diciembre de 2013].

Chaín-Revuelta, R., N. Jácome y M. Martínez (2001), "Alumnos y trayectorias. Procesos de análisis de información para diagnóstico y predicción", en Deserción, rezago y eficiencia terminal en las IES (Serie Investigaciones), México, ANUIES, <http://www.anuies.mx/servicios/d_estrategicos/libros/ lib64/8.html> [Consulta: noviembre de 2010].

Chaín-Revuelta, R. y C. Ramírez-Muro (1997), "Trayectoria escolar: la eficiencia terminal en la Universidad Veracruzana", en Revista de Educación Superior, vol. 26, núm. 102, pp. 79-97, <http://www. anuies.mx/servicios/p_anuies/publicaciones/revsup/ res102/info102.htm> [Consulta: octubre de 2015].

Cuevas, M. S. (2001), "La trayectoria escolar universitaria un acercamiento desde las habilidades básicas de pensamiento y el rendimiento académico", en Revista DIDAC. Revista de la Universidad Iberoamericana, núm. 38, pp. $45-49$.

De Allende, G. M. y J. Gómez (1989), "Bibliografía comentada sobre la trayectoria escolar", en Trayectoria escolar en la educación superior, Panorámica de la investigación y acercamientos metodológicos, México, SEP/ANUIES.

De Garay, A. y R. L. Sánchez (2012), "La modificación de la política de admisión en la UAM y los cambios en las trayectorias escolares al primer año de estudios en la Unidad Azcapotzalco", en Perfiles Educativos, vol. XXXIV, núm. 135, pp. 78-99, <http://www.scielo. org. $\mathrm{mx} /$ scielo.php? script=sci_arttext\&pid=S0 185 -26982012000100006> [Consulta: agosto de 2016].

Fernández-Pérez, J. A., A. Peña-Chumacero y F. VeraRodríguez (2006), "Los estudios de trayectoria escolar. Su aplicación en la educación media superior", en Revista de la Facultad de Filosofia y Letras, núm. 6, pp. 24-29, <https:/ / dialnet.unirioja.es / servlet/articulo? codigo $=2290466>$ [Consulta: agosto de 2013].

García, O. y C. Barrón (2011), "Un estudio sobre la trayectoria escolar de los estudiantes de doctorado en pedagogía", en Perfiles Educativos, vol. XXXIII, núm. 131, pp. 94-113, <http://www.redalyc.org/ pdf/132/13218531007.pdf> [Consulta: agosto de 2015].

González, A. (2000), Seguimiento de trayectorias escolares en la LEMO de la BUAP. Cohorte 1993, México, ANUIES (Colección Biblioteca de la Educación Superior).

González, A. (1999), Seguimiento de trayectorias escolares, México, ANUIES.

González, A., E. Castro y D. Bañuelos (201 1), “Trayectorias escolares. El perfil de ingreso de los estudiantes de Ciencias Químicas: un primer abordaje para contrastación ulterior con otras disciplinas", en Revista Latinoamericana de Estudios Educativos, vol. XLI, núms. 3 y 4, pp. 119-138, <http://www.redalyc.org/pdf/270/27022351006. pdf> [Consulta: febrero de 2015].

Gutiérrez-García, A. G., D. E. Granados-Ramos y M. G. Landeros-Velázquez(2011), "Indicadoresdelatrayectoria 
escolar de los alumnos de psicología de la Universidad Veracruzana", en Revista Electrónica Actualidades Investigativas en Educación, vol. 11, núm. 3, pp. 1-30, <http://www.redalyc.org/pdf/447/44722178009. pdf $>$ [Consulta: marzo de 2017].

Instituto Nacional para la Evaluación de la Educación (INEE) (2012), "Panorama Educativo de México 2010. Indicadores del Sistema Educativo Nacional. Educación Básica y Media Superior", <http:// www.inee.edu.mx/sitioinee 10/Publicaciones / IndicadoresEducativos/P1B109PNRMEMS2010. pdf> [Consulta: abril de 2014].

March, J. y J. Olsen (1997), El redescubrimiento de las instituciones. La base organizativa de la politica, México, Fondo de Cultura Económica.

Organización para la Cooperación y el Desarrollo Económico (OCDE) (2014), "Panorama de la educación 2014", <http://www.oecd.org/edu/Mexico-EAG2014Country-Note-spanish.pdf> [Consulta: abril de 2014].

Ponce de León, M. del S. (2003), "Guía para el seguimiento de trayectorias escolares", México, Universidad Autónoma del Estado de Hidalgo, <http://intranet. uaeh.edu.mx/DGP/pdf/2_guia_trayectoria.pdf $>$ [Consulta: marzo de 2017].

Rodríguez, A. (1997), El análisis de las trayectorias escolares como herramientas de evaluación de la actividad académica universitaria, México, ANUIES.

Sánchez-Olavarría, C. (2017), "Trayectorias profesionales docentes en posgrado: un estudio desde sus funciones", en Revista Dilemas Contemporáneos: Educación, Política y Valores, vol. 4, núm. 3, pp. 1-21, <https://www. dilemascontemporaneoseducacionpoliticayvalores. com/edici\%E2\%99\%80n-2013/ano-iv-publicacionno-3-mayo-2017/> [Consulta: diciembre 2017 ].
Sánchez-Olavarría, C. (2014), Trayectorias de egresados de ciencias de la comunicación: un estudio de caso en la Universidad del Altiplano, México, Díaz de Santos.

Sánchez, D. A., D. Alamilla y O. García (2013), "Factores que intervienen en la trayectoria escolar de la licenciatura en Derecho y Jurisprudencia en el Instituto de Ciencias Sociales y Humanidades de la UAEH”, en Edähi. Boletín Científico del Instituto de Ciencias Sociales y Humanidades, UAEH, núm. 1, pp. 1-20, <http://www.uaeh.edu.mx/ scige/boletin/icshu/n1/> [Consulta: enero de 2017].

Secretaría de Educación Pública (SEP) (2017), "Becas de Educación Media Superior", <http://www. becasmediasuperior.sep.gob.mx/> [Consulta: octubre de 2017].

SEP (2016), "Principales cifras del sistema educativo nacional 2015-2016", <http://www.planeacion.sep. gob.mx/Doc/estadistica_e_indicadores/principales_ cifras/principales_cifras_2015_2016_bolsillo_ preliminar.pdf $>$ [Consulta: junio de 2017].

SEP (2015), "Yo no abandono", <http://www.sems.gob. $\mathrm{mx} / \mathrm{swb} / \mathrm{sems}$ /yo_no_abandono $>$ [Consulta: junio de 2016].

Silas, J. C. (2013), "Acreditación, mercado y educación superior”, en Reencuentro, núm. 67, pp. 17-25, <http:// www.redalyc.org/pdf/340/34030523003.pdf> [Consulta: febrero de 2015].

Terigi, F. (2007), "Los desafíos que plantean las trayectorias escolares", Ponencia presentada en el III Foro Latinoamericano de Educación Jóvenes y Docentes. La escuela secundaria en el mundo de hoy, organizado por la Fundación Santillana en Buenos Aires, 28-30 de mayo, <http://www.ieo.edu.ar/promedu/trayescolar/ desafios.pdf> [Consulta: mayo de 2013].

\section{Cómo citar este artículo:}

Sánchez-Olavarría, César (2019), "Trayectorias escolares en el nivel medio superior: el caso de una institución pública", en Revista Iberoamericana de Educación Superior (RIES), México, UNAM-IISUE/Universia, vol. x, Núm. 28, pp. 66-81, DOI: https://doi.org/10.22201/ iisue.20072872e.2019.28.429 [consulta: fecha de última consulta]. 